\title{
NEW CLUSTERING SCHEMES FOR WIRELESS SENSOR NETWORKS
}

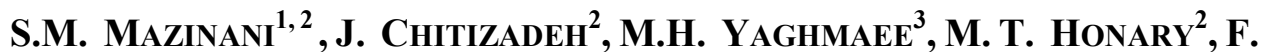 \\ TASHTARIAN ${ }^{4}$ \\ ${ }^{I}$ Dept. of Electrical and Computer Engineering, Imam Reza University, Mashhad, Iran \\ ${ }^{2}$ Dept. of Electrical Engineering, Ferdowsi University, Mashhad, Iran \\ ${ }^{3}$ Dept. of Computer Engineering, Ferdowsi University, Mashhad, Iran \\ ${ }^{4}$ Dept. of Information Technology, Islamic Azad University, Mashhad, Iran \\ \{smajidmazinani,mh_yaghmaee, tashtarian,_m.tolou,\}@gmail.com \\ chitizad@um.ac.ir
}

\begin{abstract}
In this paper, two clustering algorithms are proposed. In the first one, we investigate a clustering protocol for single hop wireless sensor networks that employs a competitive scheme for cluster head selection. The proposed algorithm is named EECS$\mathrm{M}$ that is a modified version to the well known protocol EECS where some of the nodes become volunteers to be cluster heads with an equal probability. In the competition phase in contrast to EECS using a fixed competition range for any volunteer node, we assign a variable competition range to it that is related to its distance to base station. The volunteer nodes compete in their competition ranges and every one with more residual energy would become cluster head. In the second one, we develop a clustering protocol for single hop wireless sensor networks. In the proposed algorithm some of the nodes become volunteers to be cluster heads. We develop a time based competitive clustering algorithm that the advertising time is based on the volunteer node's residual energy. We assign to every volunteer node a competition range that may be fixed or variable as a function of distance to BS. The volunteer nodes compete in their competition ranges and every one with more energy would become cluster head. In both proposed algorithms, our objective is to balance the energy consumption of the cluster heads all over the network. Simulation results show the more balanced energy consumption and longer lifetime.
\end{abstract}

KEYWORDS: wireless sensor networks, clustering, residual energy, balanced energy, single hop.

\section{INTRODUCTION}

The sensor network is a collection of small-size, low-power, low-cost sensor nodes that have some computation, communication, storage and even movement capabilities. These nodes can operate unattended, sensing the environment, generating data, processing data, and providing the data to users. With these features, sensor networks have been adopted in many pervasive computation and communication scenarios such as remote surveillance, habitat monitoring, and so on $[1,2]$. 
The deployment of wireless sensor networks in many application areas, e.g., aggregation services, requires self-organization of the network nodes into clusters. In these cases, sensors in different regions of the field can collaborate to aggregate the information they gathered. For instance, in habitat monitoring applications the sink may require the average of temperature; in military applications the existence or not of high levels of radiation may be the target information that is being sought. It is evident that by organizing the sensor nodes in groups i.e., clusters of nodes, we can reap significant network performance gains. Clustering not only allows aggregation, but limits data transmission primarily within the cluster, thereby reducing both the network traffic and the contention for the channel.

In this case, the gathered data from each node is processed locally and aggregated in a central coordinator referred to as a clusterhead $(\mathrm{CH})$ and the redundant data (if any) is omitted to provide more accurate reports about the local region being monitored. In addition, data aggregation reduces the communication overhead in the network, leading to significant energy savings. Node clustering is an efficient network organization in order to support data aggregation and it improves network lifetime [3].

Direct transmission (single hop) and hop-by-hop (multi hop) transmission are two basic communication patterns in wireless networks. It was noticed that in the case of single hop communication the furthest sensors tend to deplete their energy budget faster than other sensors. In other words in direct transmission where packets are directly transmitted to the sink without any relay, the nodes located farther away from the sink have higher energy burden due to long range communication, and these nodes may die out first. To achieve balanced energy consumption, an elegant solution is to do make the clusters' size related to the energy consumption of the cluster heads. Thus smaller clusters are needed in the further distances from the BS in order to save more energy for the cluster heads. The main contribution in this paper is to gain energy balancing through clustering in such single hop networks.

With this motivation, we propose a clustering protocol as the first proposed algorithm that employs level's total energy and their distances to BS to form clusters. We point to a competitive algorithm proposed in [4] named EECS, for selecting CHs among many other tentatively selected nodes. In the proposed algorithm that we name it EECS-M (modified EECS) some of the nodes become volunteers to be $\mathrm{CH}$ in the network. Then they start broadcasting a competition message in their pre-assigned competition range is (in contrast to EECS using a fixed competition range) a function of distance to BS. Every other volunteer node in its competitive range would quit the competition, if they have less energy.

Cluster architecture simplifies topology management and reduces the number of sensor nodes contending for the channel access. However, a $\mathrm{CH}$ drains its energy more quickly than ordinary nodes due to the more computational and operational activities. Thus, A $\mathrm{CH}$ election algorithm must be distributed, energy-efficient, and load balanced [3]. With this motivation, we also propose a clustering algorithm as the second one in this paper that employs node's residual energy for $\mathrm{CHs}$ ' selection and their distance to BS to form clusters. We point to EECS as a competitive algorithm for selecting CHs among many other tentatively selected nodes. A variable competition range, which is derived from a 
recursive formula, is chosen which is proportional to the cluster's size. As it is shown later, these unequal clusters make the load to distribute evenly on the whole network and results in more nodes' longevity. In the second proposed algorithm, the whole network's lifetime is the time until the first node in the network runs out of energy.

In fact in the second proposed algorithm, we developed a competitive clustering algorithm that uses a time based advertising procedure. Initially some of the nodes become volunteers to be $\mathrm{CH}$ in the network. Then they start advertising in the network using a timing schedule that is a function of energy. Every node that has more residual energy would start advertising sooner. Each node is also assigned a competitive range that may be fixed or variable as a function of distance to BS. Every other volunteer node in its competitive range would stop advertising, if they have less energy. Otherwise they will wait for their turn for advertising.

The remainder of this paper is outlined as follows: in section 2, we present related work on some previous well-known clustering schemes proposed in the literature. In section 3 a common model for WSNs is introduced and network's operation stages including cluster selection/formation phase and data gathering/reporting phase are described for both algorithms. Section 4 focuses on the proposed algorithms in details. Simulation results are also included in section 5.

\section{RELATED WORK}

Lots of methods and researches are dealing with energy efficient issues and to prolong the lifetime of the wireless sensor networks and in the past few years, many clustering algorithms have been proposed for ad hoc and sensor networks aiming to improve the energy efficiency. LEACH [5] is an application-specific clustering protocol that utilizes random selection and frequent rotation of $\mathrm{CHs}$ for distribution of the total load into all nodes. The clustering process involves only one iteration, after which a node decides whether to become a $\mathrm{CH}$ or not and nodes take turns in carrying the $\mathrm{CH}$ 's role. The data communication in LEACH is based on single-hop communication model. The author also proposed two variants of LEACH, which are referred to as LEACH-C (LEACHcentralized), and LEACH-F (LEACH with Fixed clusters). AROS [6] is a new version of LEACH which uses asymmetric communication with a semi-centralized clustering algorithm. The author demonstrated that AROS improves communication energy efficiency when the network size increases. HEED [7] selects $\mathrm{CHs}$ through $\mathrm{O}(1)$ time iteration according to a hybrid of nodes' residual energy and another parameter such as node proximity to its neighbors or node degree.

The author in [4] presented EECS, which is a novel approach to distribute the CHs uniformly across the network through a localized single hop communication with little overhead. A competitive algorithm is suggested for $\mathrm{CHs}$ selection phase and a fixed competition range is specified to each volunteer node. A weighted cost function is also introduced to manage the number of cluster members. Every node, which finds a more powerful node in its competition set, will give up the competition immediately and broadcast its QUIT acknowledgment message. Any node that finds itself more powerful than the others in its competition radius will introduce itself as a $\mathrm{CH}$ and broadcast its 
advertisement message. The message complexity in this algorithm makes trouble in the dense networks for having too many nodes competing for being $\mathrm{CH}$. A similar approach is performed in [8] where the authors applied a variable competition radius to the tentative nodes. An energy balancing criterion for every $\mathrm{CH}$ is used to derive a recursive equation for its competition range based on the nodes distance to the BS.

In [9], several energy-efficient communication protocols have been proposed based on power control and load balancing, aiming at even distribution of the residual energy of the sensors and thus prolonging network lifetime. Dagher, et. al. [10] presented a theory for maximizing the lifetime of multihop WSN. An optimal centralized solution was presented in the form of an iteration algorithm. In [11], a taxonomy and general classification of published clustering schemes was presented. The authors surveyed different clustering algorithms for WSNs. In [12], an investigation about cluster size and the number of cluster heads in the region was achieved when all the devices in a WSN are deployed randomly.

In this paper, since we are not to design an efficient MAC layer, an ideal simple MAC layer is recommended that is collision-free and uses a TDMA schedule for nodes' data communication. Our goal is to balance the energy consumption in the whole network in such a way that network's lifetime is increased.

\section{SYSTEM MODEL}

To illustrate the impact of the physical limits of sensor networks on the design of our algorithms we briefly discuss related wireless network model, which highly depends on the application. We consider a network with characteristics as below:

- The sensor nodes are homogenous and they are uniformly distributed in a square field.

- The sink and the sensor nodes are assumed to be static once deployed.

- The sink node or Base Station (BS) is sited outside of the square field.

- All sensor nodes are able to set their power transmission according to the distance to the destination.

- Sensor nodes are assigned a unique ID and they are fully synchronized via a synchronization beacon broadcasted from the BS at the beginning of every round.

\section{A. Network's Operation and Data Gathering Model}

For most data gathering applications, the sensors usually operate in a low-duty-cycle mode. The interval between one duty cycle to the next may be several minutes, hours even days. This characteristic motivates the utilization of periodical sleeping to conserve energy. Each $\mathrm{CH}$ acts as a local coordinator of data transmission in the cluster. It sets up a TDMA schedules for all the cluster's nodes to ensure that there is no collisions among data messages. This schedule also allows the radio component of relative nodes to be turned off at all the times except during their transmit time [5]. The data reporting process, which sometimes is called "steady state phase", is divided into rounds. In each round, any non- $\mathrm{CH}$ nodes in a cluster send their raw data obtained from sensing environment directly 
to their respective CHs. The nodes are also supposed to embed their residual energy information in the same packet. This information would be useful to compute the average network's energy in the BS.

The CHs nodes are responsible for coordinating the members in their clusters and communicate with the sink node on behalf of their clusters and via a single hop communication. In addition, $\mathrm{CHs}$ aggregates the received data from its members with its own gathered data to compress the amount of data, which is to be sent to the BS. The data aggregation increases the performance of the network in terms of communication cost. However, the optimality of performing data aggregation is beyond the scope of this paper. At the end of each round, the clustering phase will restart and the new $\mathrm{CHs}$ are selected for the next round.

\section{B. Energy Consumption Model in Single Hop Networks}

In our model, we assume that each sensor can intelligently choose the transmission power based on the link distance. This is true in typical sensor node implementations. A simple model is described in [5] where each node dissipates energy to run the radio electronics and power amplifier in transmitter side. The power attenuation is a function of the distance between transmitter and receiver. There is a cross over distance that can be simply used to model the propagation loss. Thus, to transmit a l-bit message to a distance $\mathrm{d}$, the radio expends:

$$
E_{T x}(d)= \begin{cases}l E_{\text {elect }}+l \varepsilon_{\text {freespace }} d^{2} & d<d_{\text {crossover }} \\ l E_{\text {elect }}+l \varepsilon_{\text {multipath }} d^{4} & d \geq d_{\text {crossover }} .\end{cases}
$$

where the $E_{\text {elect }}$ is the energy required for running the electronic circuits. $\varepsilon_{\text {freespace }}$ and $\varepsilon_{\text {multipath }}$ are two parameters which depend on the noise figure and the required SNR for proper signal detection at the receiver. It can also be written in a more general from as:

$$
E_{T x}(d)=p+q d^{\alpha}
$$

where $p$ and $q$ are constants related to node energy dissipation to run the radio electronics and power amplifier in transmitter and $\alpha$ is the path loss factor.

Besides, there are two more sources of energy consumption of the $\mathrm{CHs}$. Each $\mathrm{CH}$ consumes energy for receiving data from adjacent nodes or cluster members and fuses it into a single packet that is outlined by:

$$
E_{r f}=m l E_{\text {elect }}+E_{B F}
$$

where in both equations, $m$ is the number of cluster's members and EBF is the computed energy for beam forming data aggregation [5]. 


\section{THE PROPOSED ALGORITHMS}

In this paper, we propose two clustering algorithms which result in more balanced energy consumption and longer lifetime.

\subsection{Details of First Algorithm (EECS-M)}

As discussed previously, any detected event must be reported to the BS in the form of data packets. In some applications such as fire detection, the event must be reported as soon as possible and too much latency is unacceptable. The recommended communication model for such networks is single hop communication. Nevertheless, further nodes consume more energy for data reporting.

\section{A. Determining the Length of Levels}

Figure 1 depicts a simple network which is divided into three radial levels with the BS at the center. The clusters located in the further levels seem to be smaller than the closer clusters. Thanks to having more distance to the $\mathrm{BS}$, the $\mathrm{CH}$ needs to spend more power to send its own packet. Having smaller cluster formed with less number of members, the $\mathrm{CH}$ can save most of its energy for data communication to the BS. On the other hand, since the closer $\mathrm{CH}$ nodes do not consume a large amount of energy for data communication to the $\mathrm{BS}$, they are able to form larger clusters to cover more area and support more number of members.

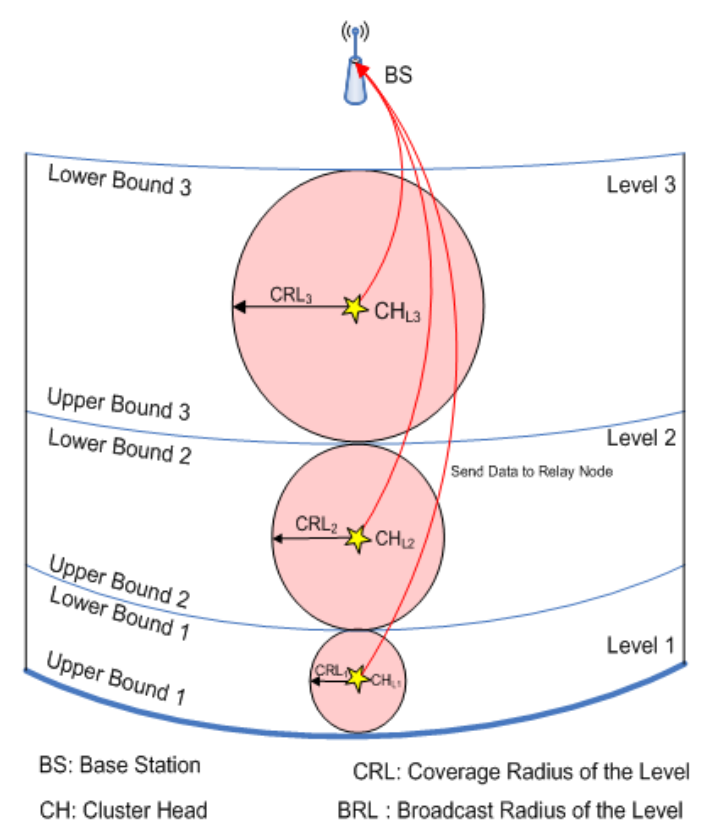

Fig. 1: Radial regions (levels) with the BS at the center.

The contribution is to gain energy balancing for each individual level. The total energy consumption of the $i^{\text {th }}$ level is the sum of the consumed energies of every single cluster in that level. Considering $k$ number of clusters in a level, the total level's energy is: 
$E_{\text {Level }}^{i}=k \times E_{\text {cluster }}$

Where $E_{\text {cluster }}$ (the total energy consumed in each cluster) is composed of the total energy consumed by the member nodes and the energy consumed by the $\mathrm{CH}$ itself as follows:

$E_{\text {cluster }}=E_{C H}+E_{C M}$

Where $E_{C H}$ and $E_{C M}$ are the CH's energy consumption and cluster members' energy consumption respectively. The $\mathrm{CH}$ receives all the packets from the members and fuses it into a single packet and forwards to the BS. Considering $\rho$ as the nodes density and a circular region for the cluster with the coverage radius $C R L$, the energy consumed by the $\mathrm{CH}$ is as follows:

$$
E_{C H}=\pi \cdot C R L^{2} \cdot \rho \cdot\left(E_{r f}\right)+p+q d_{t o B S}^{4}
$$

Where $d_{t o B S}$ is the total distance from the $\mathrm{CH}$ to the BS. If the network's width is named $y$ and the distance of the BS to the closest edge of the network is called $R_{0}$ (see Fig. 2), we have:

$$
d_{\text {toB S }}=y+R_{0}-C R L
$$

The cluster members' energy consumption can be derived as bellow:

$$
E_{C M}=\pi \cdot C R L^{2} \cdot \rho \cdot\left(p+q d_{t o C H}^{2}\right)
$$

Generally speaking the expectation of nodes' distance to the $\mathrm{CH}$ must be applied in Eq. (8). In [5] it is derived as follows:

$$
\begin{aligned}
E\left[d_{t o C H}^{2}\right] & =\iint\left(x^{2}+y^{2}\right) \rho(x, y) d x d y \\
& =\int_{0}^{2 \pi} \int_{0}^{C R L} \rho r^{3} d \theta d r \\
& =\rho \cdot 2 \pi \cdot \frac{C R L^{4}}{4}
\end{aligned}
$$

Where in the last step it is assumed that the node's density, $\rho$, is independent of the physical positions. Inserting Eq. (6) and Eq. (8) into Eq. (5) and the result into Eq. (4), the total energy consumption of the $i^{\text {th }}$ level is: 


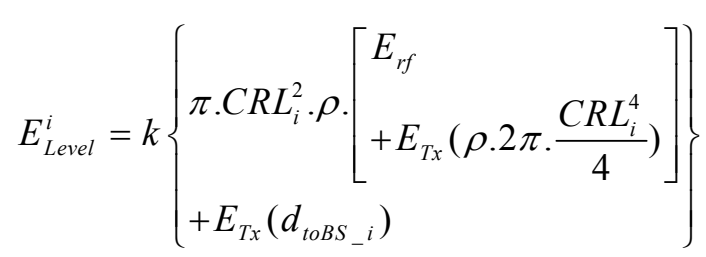

Where $k$ is the number of clusters in that level:

$$
k=\frac{2 \cdot C R L_{i} \cdot y}{\pi \cdot C R L_{i}{ }^{2}}
$$

Also $d_{\text {toBS } \_}$is derived as follows:

$$
d_{\text {toBS } S_{-} i}=y+R_{0}-2 \sum_{j=1}^{i-1} C R L_{j}-C R L_{i}
$$

In this equation there are two unknowns, $E_{\text {level }}^{i}$ and $C R L_{i}$. The energy balancing criterion in this article is the equality of levels' total energy consumption. Since the furthest nodes from the BS are supposed to die faster, we compute the minimum of the first level's total energy consumption and equal this to the other levels'. By this way we get rid of the unknown $E_{\text {level }}^{i}$.

At this time considering $i=1$ in Eq. (10) we get an equation for the first level's total energy consumption as a function of $C R L_{1}$. Having this equation minimized, the derivation with respect to $C R L$ must be vanished. It is easy to show that Eq. (10) has minimum and the derivation has a single acceptable result in the feasible range for the last level.

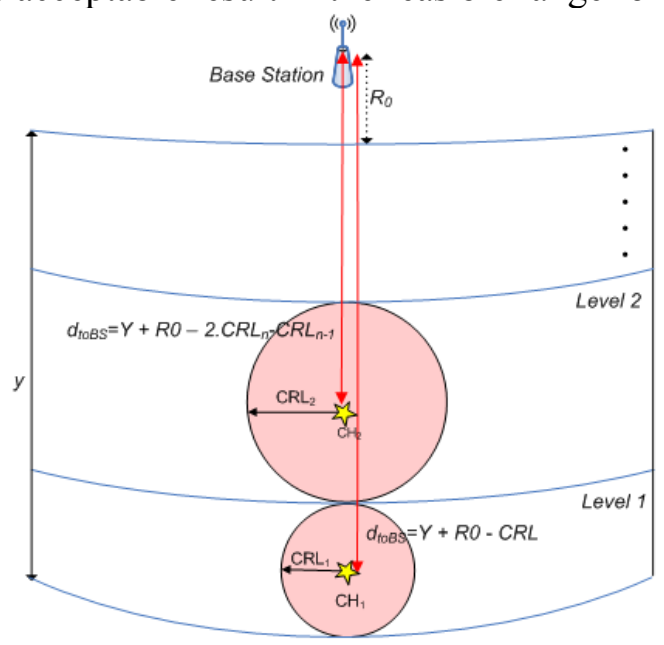

Fig. 2: Clusters grow up by enclosing the BS.

Using the obtained result, the minimum of the first level's total energy consumption can be reached and it is considered equal to the other level's total energy consumption. Therefore according to Eq. (10) $E_{\text {level }}^{i}$ is known and only the $C R L_{i}$, is unknown which can 
be computed easily for other levels. In Fig. 2, it is obvious that the clusters grow up by enclosing to the BS.

\section{B. Competitive Clustering Algorithm}

This section describes the CHs' selection and cluster formation phase. In this stage we refer to [4] for the competition phase among those tentatively selected nodes. In this clustering scheme, the tentative nodes compete for being final $\mathrm{CHs}$. These nodes become tentative with an equal probability $T$ and they broadcast a competitive message in their competition radius which is assumed to be fixed in EECS. This message contains the node's $I D$, its residual energy and its competitive range, $R_{\text {competition }}$. If there is any other tentative node in this radius it would hear this message. Any node that finds itself more powerful than the others in its competition radius will introduce itself as a $\mathrm{CH}$ and broadcast its advertisement message.

The main difference of the proposed algorithm and EECS is in the competition radius which was considered to be fixed in EECS. In this algorithm we consider that every tentative node competes in its respective level's CRL. As we can see the competition range of each level increases as the region's distance from the BS decreases. This means the smaller clusters with fewer nodes joining them is expected to be formed in far distances to BS.

\subsection{Details of Second Algorithm}

This section describes the CHs' selection and cluster formation phase. In our purposed clustering scheme, we assumed that the nodes, whose residual energies are more than the average network's energy, are more proper to become a $\mathrm{CH}$ at each round. For this purpose, we need to take the advantage of the hello message that is broadcasted from the $\mathrm{BS}$ at the beginning of every round for nodes synchronization. This hello message contains the average network's energy that is computed in the BS. Remember that we assumed that every node is supposed to send its residual energy to its respective $\mathrm{CH}$, and $\mathrm{CH}$ is supposed to embed the average cluster's energy in the header of the aggregated data packet and forward it to the BS.

The next step is the competition stage where the tentative nodes compete for final CHs. In the previous proposed algorithm, EECS, the nodes has to broadcast a competitive message in their competition radius and if there is any other tentative node in this radius would hear this message. Any node that finds itself more powerful than the others in its competition radius will introduce itself as a $\mathrm{CH}$ and broadcast its advertisement message. The message complexity in this algorithm makes trouble in the dense networks for having too many nodes competing and negotiating too many packets for being $\mathrm{CH}$.

We omitted the negotiation in the competition phase and replaced it with a timing schedule for advertising nodes. In this algorithm each tentative node will broadcast its advertisement message across the network based on a timing schedule as bellow:

$$
T_{\text {Advertisement }}=\left(\frac{E_{\text {initial }}-E_{\text {residual }}}{E_{\text {initial }}}\right) \cdot t_{0 .}
$$


Where $E_{\text {initial }}$ and $E_{\text {residual }}$ are the initial and residual energy of the volunteer node respectively and $t_{0}$, is the maximum waiting time for advertisement and is a predefined parameter. It is obvious that the node with more residual energy will advertise itself sooner.

In the advertising packet the node's $I D$, its residual energy and competitive range, $R_{\text {competition }}$ are embedded. Suppose that $S_{i}$ is a tentative node that is a volunteer to become a $\mathrm{CH}$. The goal is to remove any tentative node $S_{j}(j \neq i)$ that is in $S_{i}$ 's competition set and its energy is less than $S_{i}$ 's residual energy. If $S_{j}$ hears the advertisement and it determines that it has less residual energy than $S_{i}$, it stops waiting for advertising itself and quits the competition immediately and goes to sleep mode like other ordinary nodes. Otherwise it waits for its turn to advertise and starts advertising if there is no any heard advertising message. The low message complexity in this approach is obvious since no extra negotiation is needed between the competing nodes. In the simulation section we will demonstrate the effectiveness of using timing schedule on the network's lifetime.

In the first step we consider a fixed competition range just like EECS. Then we refer to [8] on deriving a competitive range as a function of distance to BS for a divided network into equal-length radial levels with the BS at the center as the following:

$$
R_{i+1}=\sqrt{R_{i}^{2}-\frac{\left(2 \alpha d_{i}+\alpha^{2}\right)}{k}}
$$

Where according to [8] $R_{i}$ is the competition range of the $\mathrm{CH}$ in the $\mathrm{i}^{\text {th }}$ level. The parameter $\alpha$ and $d_{i}$ are the region increment and the region distance to the BS respectively and the parameter $k$ is a constant dependent to the network's primarily settings. By this equation, the competition range of each $\mathrm{CH}$ is derived based on its distance to the $\mathrm{BS}$ and the obtained competition range for the previous level. As we can see the competition range of each level decreases as the region's distance from the BS increases. This means the smaller clusters with fewer nodes joining them is expected to be formed in far distances to $\mathrm{BS}$. Therefore, less energy for receiving data is consumed and $\mathrm{CH}$ can save majority of its energy for data transmission to the BS.

We evaluated the network's efficiency in the sense of lifetime using a variable competition range and a timing schedule together. The results are brought with details in the next section.

\section{SIMULATION AND RESULTS}

The performance of proposed algorithms is evaluated via MATLAB.

\section{A. EECS-M Evaluation}

In this section, EECS-M is evaluated. The simulated network has the characteristics described in section 2. Other simulation parameters are listed in Table. 1. The simulations are performed in fair situations and the results are compared with the EECS algorithm. 
In Fig. 3 the network's lifetime is depicted in two scenarios in the sense of the number of alive nodes. The EECS-M shows longer lifetime with respect to EECS. More fairness in load distribution is obtained in our proposed algorithm by using this variable competition range (variable cluster sizes) for each level. 
TABLE 1: SimULATION PARAMETERS

\begin{tabular}{|c|c|}
\hline Parameter & Value \\
\hline Network size & $300 \times 300 \mathrm{~m}^{2}$ \\
\hline BS location & $150,500 \mathrm{~m}$ \\
\hline \multirow{2}{*}{ Number of Sensors } & 400 (scenario1) \\
\hline & 800 (scenario2) \\
\hline Initial Energy & $0.5 \mathrm{~J}$ \\
\hline $\mathrm{E}_{\text {elec }}$ & $50 \mathrm{~nJ} / \mathrm{bit}$ \\
\hline$\varepsilon_{\mathrm{fs}}$ & $10 \mathrm{pJ} / \mathrm{bit} / \mathrm{m}^{2}$ \\
\hline$\varepsilon_{\mathrm{mp}}$ & $0.0013 \mathrm{pJ} / \mathrm{bit} / \mathrm{m}^{4}$ \\
\hline $\mathrm{E}_{\mathrm{BF}}$ & $5 \mathrm{~nJ} / \mathrm{bit} /$ singnal \\
\hline Data Packet Size & 4000 bits \\
\hline Control Packet Size & 32 bits \\
\hline $\mathrm{d}_{0}$ & $87 \mathrm{~m}$ \\
\hline $\mathrm{T}$ & 0.2 \\
\hline
\end{tabular}

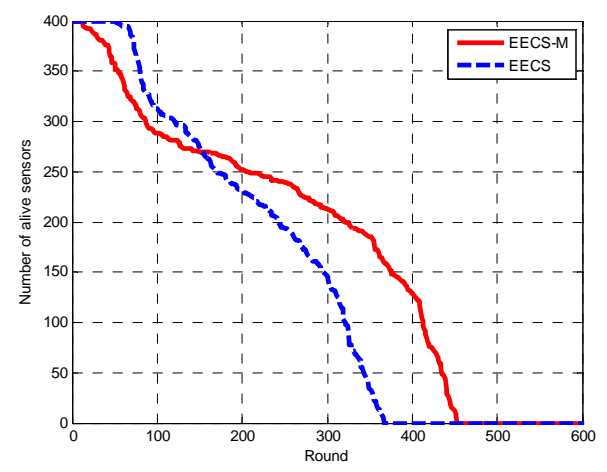

(a)

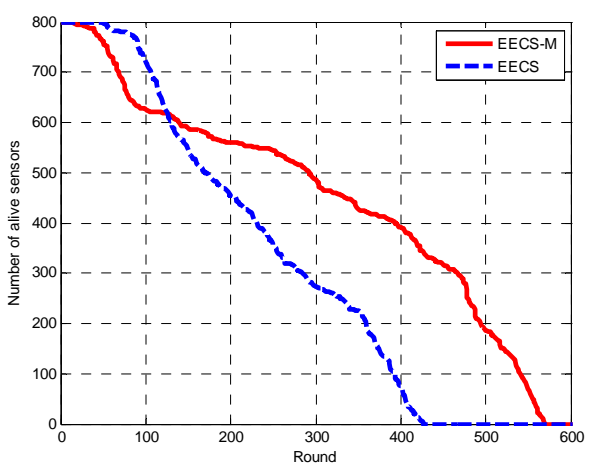

(b)

Fig. 3: Number of alive nodes. (a) scenario1 (400 nodes), (b) scenario2 (800 nodes).

Measuring the total network' energy consumption is useful to compare the three cases for the whole lifetime (Fig. 4). The lower steep in the figure shows more fairness in energy distribution. It implies that the nodes consume their energy much slower than other case. EECS-M shows more efficient energy consumption than EECS in the both scenarios. This is due to using unequal clusters based on their distances to the BS.

Another useful parameter for efficiency evaluation is the total number of data packets received at the $\mathrm{BS}$. It is assumed that each $\mathrm{CH}$ forwards a single packet to the $\mathrm{BS}$ at each round of network's lifetime. Then one may guess this parameter is only related to the number of clusters formed in the network. However, the total number of packets received at the BS is also related to the nodes average lifetime. 


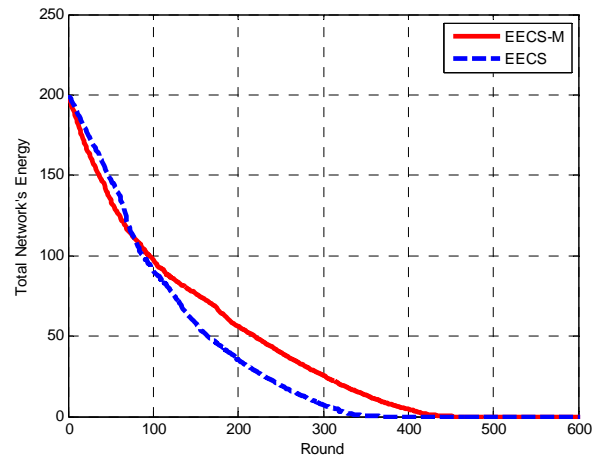

(a)

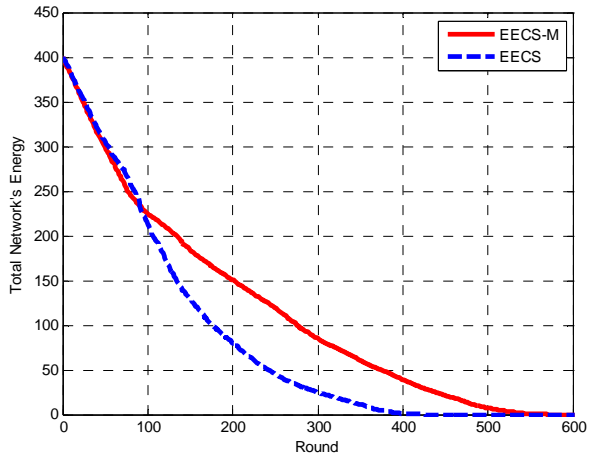

(b)

Fig. 4: Total network's energy. (a) scenario1 (400 nodes), (b) scenario2 (800 nodes).

If there are too many clusters formed in the network without considering their distance to the BS, the energy of the $\mathrm{CH}$ nodes would drain so quickly. Even if they can forward many packets to the BS, they will not last long enough to do so. Fig. 5 illustrates the total number of data packets received in each round of network lifetime. It is obviously clear that the proposed clustering algorithm delivers more data packets in both scenarios.

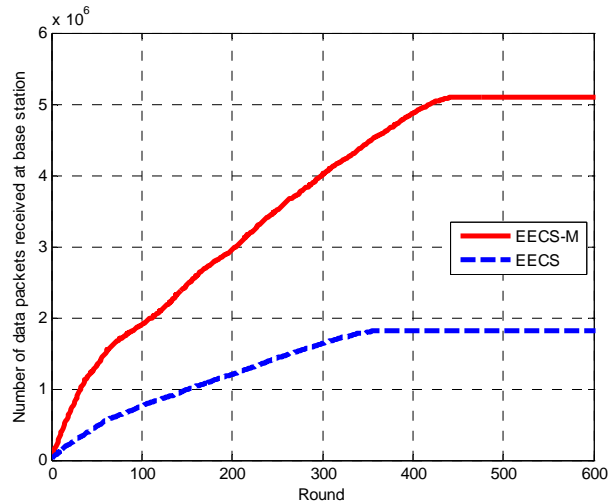

(a)

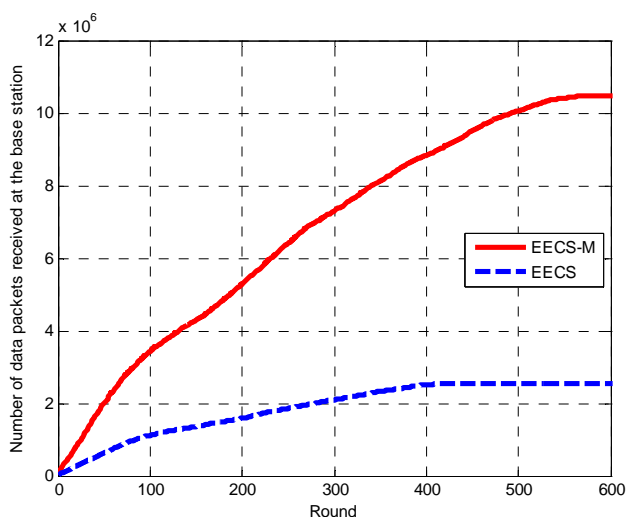

(b)

Fig. 5: Total number of data packets received at the BS.

(a) scenario1 (400 nodes), (b) scenario2 (800 nodes).

Useful numerical results are brought in table. 2. The number of levels formed using Eq. (12) is three levels for 400 nodes and four levels for 800 nodes. In both cases the FND (first node's death) in EECS-M is sooner than EECS. However the LND (last node's death) is quite longer in EECS-M with respect to EECS. The TNSP (total number of sent packets to the BS) values are interestingly larger in both cases for EECS-M that is5.1 million packets against 1.8 million in scenario 1 and so on. 
Table 2: Numerical results.

\begin{tabular}{|l|c|c|c|c|}
\hline & EECS-M & EECS & EECS-M & EECS \\
\hline \#Nodes & 400 & 400 & 800 & 800 \\
\hline \# Levels & 3 & 1 & 4 & 1 \\
\hline CRL4 & - & 133.35 & 54.65 & 112.13 \\
\hline CRL3 & 71.81 & - & 54.06 & - \\
\hline CRL2 & 70.23 & - & 51.82 & - \\
\hline CRL1 & 49.70 & - & 39.49 & - \\
\hline FND & 5 & 51 & 9 & 42 \\
\hline LND & 453 & 367 & 570 & 427 \\
\hline TNSP & $5.1 \mathrm{e}+6$ & $1.8 \mathrm{e}+6$ & $1.04 \mathrm{e}+7$ & $2.5 \mathrm{e}+6$ \\
\hline
\end{tabular}

\section{B. The Second Algorithm Evaluation}

In this section, the second algorithm is evaluated. The simulated network has the characteristics described in section 2. Other simulation parameters are listed in Table. 3. In the figures, VCR stands for "variable competition range" and TS stands for "Timing schedule".

We simulated the network for two different cases. In the first step we apply the variable competition range derived via the recursive equation as (14) with the $\mathrm{R}_{0}$ equal to 25 on the proposed algorithm EECS. Then we employ the timing schedule and the variable competition range together. In both cases we evaluated the networks lifetime in the sense on first/last node death. The results are depicted in Fig. 6 compared with EECS with no timing schedule and a fixed competition range. We also employed the cost function described in EECS choosing the more proper $\mathrm{CHs}$ for each non- $\mathrm{CH}$ node in all the cases.

In Fig. 6 it is obvious that the timing schedule improves the efficiency in the sense of lifetime. The EECS algorithm uses a competition phase at the beginning of each round. The number of packets that has to be negotiated in this phase is quite high. Thus the nodes lose their energy at the beginning of each round. Applying the variable competition range results in an improvement in the lifetime. However this is not the case. Employing the time schedule and the variable competition range together in the algorithm shows a better improvement in network's lifetime. This is due to the better energy balancing and more fairness in load distribution by using this variable competition range. Nevertheless the timing schedule makes better energy efficiency in the setup phase of the algorithm. In Fig. 7 and Fig. 8 the same result is illustrated with the focus on the time of the first and last 
node's death in the three algorithms respectively. The improvement in the lifetime is more visible in these figures.

TABLE 3: SIMULATION PARAMETERS.

\begin{tabular}{|l|l|}
\hline \multicolumn{1}{|c|}{ Parameter } & Value \\
\hline Network size & $100 * 100 \mathrm{~m}$ \\
\hline BS location & $50,200 \mathrm{~m}$ \\
\hline Number of Sensors & 400 \\
\hline Initial Energy & $0.5 \mathrm{~J}$ \\
\hline $\mathrm{E}_{\text {elec }}$ & $50 \mathrm{~nJ} / \mathrm{bit}$ \\
\hline$\varepsilon_{\mathrm{fs}}$ & $10 \mathrm{pJ} / \mathrm{bit} / \mathrm{m}^{2}$ \\
\hline$\varepsilon_{\mathrm{mp}}$ & $0.0013 \mathrm{pJ} / \mathrm{bit} / \mathrm{m}^{4}$ \\
\hline $\mathrm{EDA}^{2}$ & $5 \mathrm{~nJ} / \mathrm{bit} / \mathrm{singnal}$ \\
\hline $\mathrm{Data}$ Packet Size & $4000 \mathrm{bits}$ \\
\hline $\mathrm{d}_{0}$ & $87 \mathrm{~m}$ \\
\hline $\mathrm{t}_{0}$ & $0.008 \mathrm{~s}$ \\
\hline $\mathrm{R}_{0}$ & 25 \\
\hline
\end{tabular}

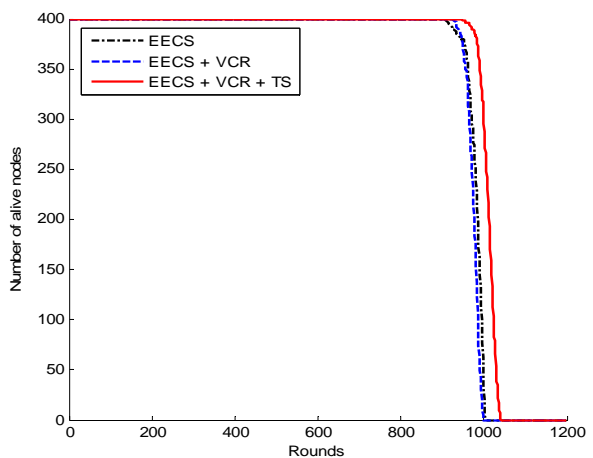

Fig. 6: Number of alive nodes.

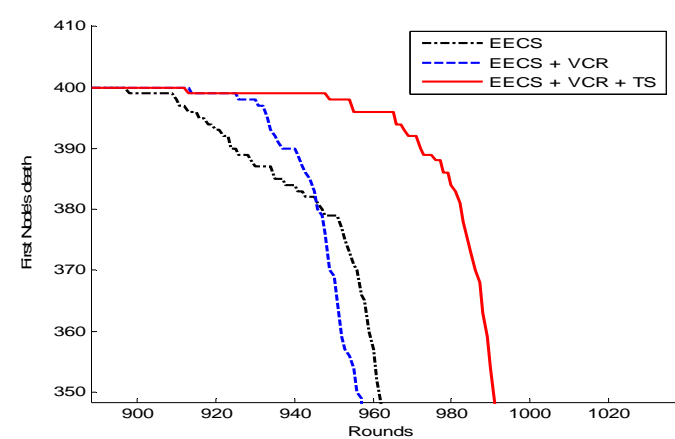

Fig.7: Network lifetime. First node's death time 


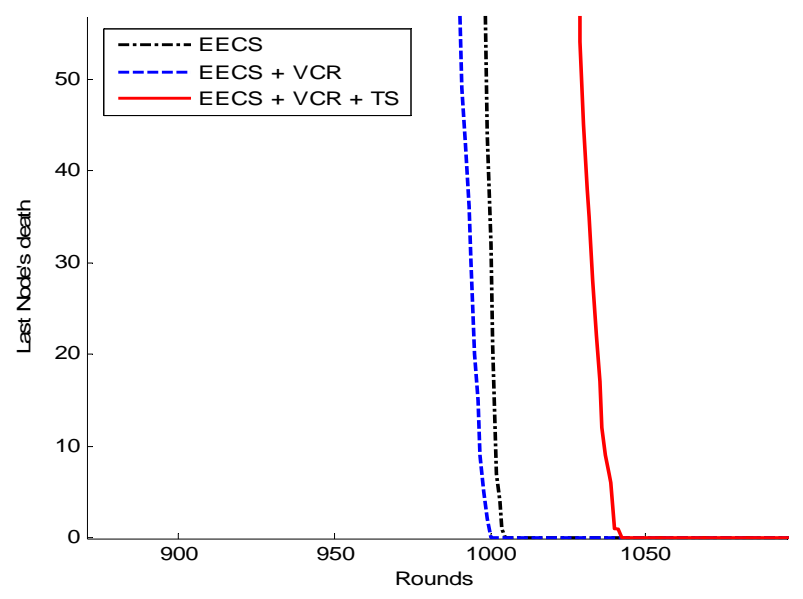

Fig. 8: Network lifetime. Last node's death time.

Measuring the total network' energy consumption is useful to compare the three cases for the whole lifetime (Fig. 9). The lower steep in the figure shows more fairness in energy distribution and longer lifetime. In this figure the competitive algorithm with timing and variable competition range shows a lower steep in decreasing the total energy of the network. It implies that the nodes consume their energy much slower than two other cases. Considerable amount of energy is consumed in EECS for competition setup phase.

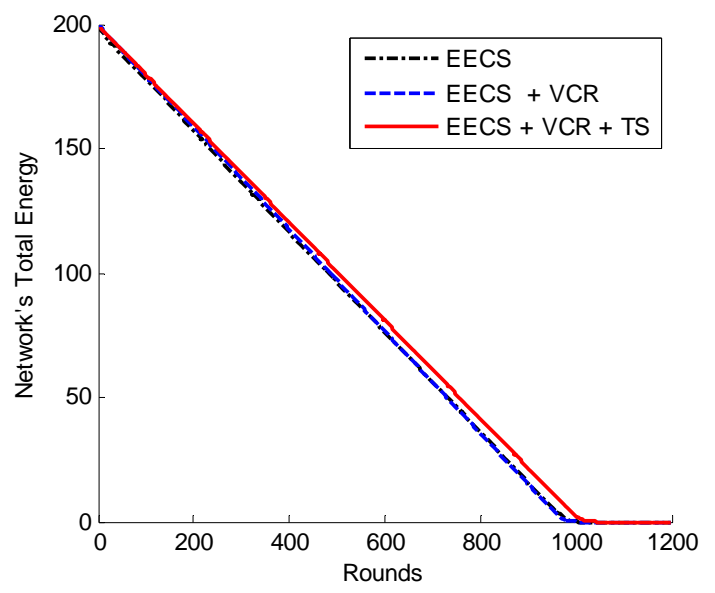

Fig. 9: Network energy consumption.

In the next step, the network is simulated for 100 independent distributions of sensor nodes. The simulation is done in fair situations for our model employing a variable/fixed competition range on the EECS algorithm and also using the timing schedule and the variable range together. A fixed competition range $R_{\text {opt }}=25$ that depends on the optimum number of clusters in the network is proposed for EECS. Figure 10 illustrates the result of network's lifetime comparison in the sense of first nodes death for 100 independent simulations. It is clear that $1.5 \%$ is achieved in applying a variable competition range on 
EECS. However the improvement in lifetime becomes $10 \%$ in using the timing schedule and the variable competition range together in the proposed algorithm.

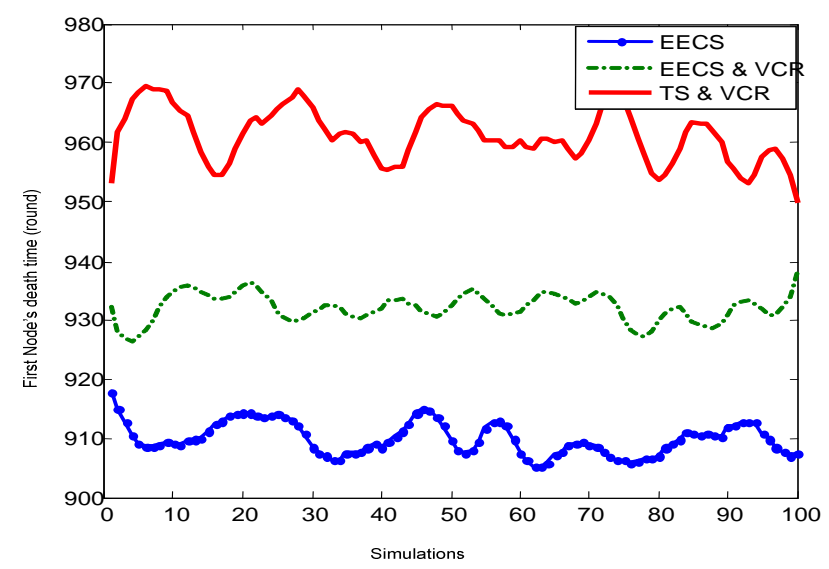

Fig. 10: Network's lifetime (first node's death) in 100 different simulations.

\section{CONCLUSION}

In this paper, we reviewed the energy balancing technique in wireless sensor networks via clustering. Energy consumption of each $\mathrm{CH}$ in such a single hop network depends on its distance to BS and the number of cluster members. In the first proposed algorithm, we provide an extension of a well-known clustering protocol that uses a competitive algorithm to form clusters. In contrast to EECS, EECS-M used unequal competition ranges of tentative $\mathrm{CH}$ nodes, which are derive as a functions of node's distance to BS. In the proposed algorithm we initially found the optimum length for the first (furthest) level in the network. The reason was that these nodes are supposed to die sooner than any other nodes closer to the BS. Then assuming equal energy consumption for every subsequent level, we found the optimum length of that level and also the number of clusters. The main difference between EECS-M and EECS is that EECS uses a fixed competitive range for tentative nodes. However, EECS-M employs the levels' total energy consumption to find the competition ranges. The proposed algorithm EECS-M prolongs the network lifetime due to more energy efficiency obtained from using the unequal clusters. The acceptable results such as longer lifetime and larger amount of received data packets in the whole network lifetime prove the effectiveness of this proposed algorithm in the sense of energy efficiency.

In the second proposed algorithm, we again provide an extension of EECS and our previous proposed algorithm in [8] that uses a competitive algorithm for determining competition range. In contrast to EECS, our model used unequal competition ranges of tentative $\mathrm{CH}$ nodes, which are recursively functions of node's distance to BS. This selection forms unequal cluster size in the whole network. Every $\mathrm{CH}$ node that is far away from the BS would form a smaller cluster with fewer members and can save most of its energy for data transmission. In the second proposed algorithm, timing schedule based advertising instead of the negotiation between the volunteer nodes is applied. This schedule was a function of nodes energy. The node with more residual energy would 
advertise itself sooner. Any node that heard the advertisement and it determined that it had had less residual energy would stop waiting for advertising itself and it would quit the competition. Otherwise it would wait for its turn to advertise and it started advertising if there have not been any heard advertising messages. We simulated the network for the ordinary algorithm EECS and our proposed timing based algorithm with/without a fixed competition range. In the first step we applied the timing schedule on the proposed algorithm EECS with the fixed competition range. Then we employed the variable competition range derived via the recursive equation as Eq. (14). In both cases we evaluated the networks lifetime in the sense on first/last node death. Employing the time schedule and the variable competition range together in the algorithm shows a better improvement in network's lifetime. This is due to the better energy balancing and more fairness in load distribution and low energy drainage in the setup phase for competition negotiation. Besides the competitive algorithm with timing and variable competition range shows a lower steep in decreasing the total energy of the network. It implies that the nodes consume their energy much slower than two other cases since considerable amount of energy is consumed in EECS for competition setup phase.

\section{REFERENCES}

[1] F. Ye, H. Luo, J. Cheng, S. Lu, and L. Zhang, "A Two-Tier Data Dissemination Model for Large-scale Wireless Sensor Networks", ACM International Conference on Mobile Computing and Networking (MOBICOM'02), pp. 148-159, September 2002.

[2] G. Wang, G. Cao, T. La Porta, and W. Zhang, "Sensor relocation in mobile sensor networks", IEEE INFOCOM, March 2005.

[3] O. Younis, M. Krunz and S. Ramasubramanian, "Node clustering in wireless sensor networks: recent developments and deployment challenges", IEEE Transactions on Networking, vol. 20, Issue. 3, pp.20-25, 2006.

[4] M. Ye, C. F. Li, G. H. Chen, and J. Wu, "EECS: An Energy Efficient Clustering Scheme in Wireless Sensor Networks", in Proceedings of IEEE Int'l Performance Computing and Communications Conference (IPCCC), pp. 535-540, 2005.

[5] W. Heinzelman, “Application-specific protocol architectures for wireless networks", Ph.D. dissertation, Mass. Inst. Technol., Cambridge, 2000.

[6] J. Neander, E. Hansen, M. Nolin, M. Bjorkman, "Asymmetric Multihop Communication in Large Sensor Networks", 1st International Symposium on Wireless Pervasive Computing, pp. $1-7,16-18$ Jan. 2006

[7] O. Younis and S. Fahmy, "HEED: a hybrid, energy-efficient, distributed clustering approach for ad hoc sensor networks", IEEE Transactions on Mobile Computing, vol. 3, no. 4, pp. $366-$ 379, Oct.-Dec 2004.

[8] M. Tolou Honary and J. Chitizadeh, "Lifetime prolonging of Wireless Sensor Networks via a recursive clustering algorithm", Proc. of third IEEE International Conference in Central Asia on internet the Next generation of mobile, wireless and optical communications networks, ICI 2007, Tashkent.

[9] Y. Wang, H. Wu, R. Nelavelli, and N. F. Tzeng, "Balance based energy-efficient communication protocols for wireless sensor networks", In Proceedings of the International Workshop on Wireless Ad hoc and Sensor Networks (WWASN2006), 2006. 
[10] J. C. Dagher, M. W. Marcellin, and M. A. Neifeld, A theory for maximizing the lifetime of sensor networks, IEEE Trans, on communications, vol. 55, pp. 323-332, Feb. 2007.

[11] A. A. Abbasi, M. Younis, "A survey on clustering algorithms for wireless sensor networks", ELSEVIER on computer communication, vol. 30, pp.2826-2841, June 2007.

[12] C. Sevgi, A. Kocyigit, "On determining cluster size of randomly deployed heterogeneous WSNs", IEEE communications letter, vol. 12, no. 4, April. 2008. 